\title{
Effect of pre-operative statin administration on delirium rate after coronary artery bypass grafting (CABG): a prospective cohort study
}

\author{
Mitra Golmohammadi ${ }^{1}$, Zahra Yekta ${ }^{2}$, Behnam Askari $^{3}$, Sepideh Rahnema ${ }^{4}$
}

${ }^{1}$ MD., Anesthesiologist, Assistant Professor, Department of Anesthesiology, Faculty of Medicine, Urmia University of Medical Sciences, Urmia, Iran

${ }^{2}$ MD of Community Medicine and Preventative Medicine, Professor, Department of Community Medicine, Faculty of Medicine, Urmia University of Medical Sciences, Urmia, Iran

${ }^{3}$ MD., Cardiac Surgeon, Assistant Professor, Department of Cardiovascular Surgery, Faculty of Medicine, Urmia University of Medical Sciences, Urmia, Iran

${ }^{4}$ MD., Anesthesiology Resident, Department of Anesthesiology, Faculty of Medicine, Urmia University of Medical Sciences, Urmia, Iran

Type of article: Original

\begin{abstract}
Background: Statins have recently been suggested to exert protective cerebral effects in patients undergoing cardiac surgery.

Objective: The aim of this study was to determine the association between pre-operative statin administration and the rate of post-operative delirium (POD) in patients undergoing coronary artery bypass grafting (CABG).

Methods: This prospective cohort study was conducted at Seyedoshohada Hospital (Urmia, Iran) from June 2017 through April 2018. Patients, before undergoing CABG may have two different approaches by clinicians; administrating statins vs. those who do not receive statins. Participants in this study were selected among each of the two approaches. Therefore, Group A ( $\mathrm{n}=200)$ (exposure group) received statins $10 \mathrm{mg}$ or $20 \mathrm{mg}$ in the preoperative period and Group B ( $\mathrm{n}=200)$ did not receive statins before admission to hospital. During their intensive care unit (ICU) stay, Confusion Assessment Method (CAM) was performed every 8 hours to assess delirium. The data were analyzed by STATA version 13. The rate of POD were determined across the groups using independent samples t-test and Chi-square tests. Study covariates were adjusted and the association between the outcome of delirium incidence and clinical interventions was examined using multiple logistic regression.

Results: Demographic data was similar between the two groups. Of the four hundred patients analyzed, 57 patients $(14.5 \%)$ had delirium at some time during their cardiovascular ICU stay; nine $(4.5 \%)$ in the patients group with use of statins and forty-eight (24\%) in the patients group without use of statins had POD. There was a significant difference in the occurrence of delirium across the two groups $(\mathrm{p}=0.001)$. Incidence of POD in patients $\geq 60$ years in Group A was significantly lower than in the patients in Group B, $4.2 \%$ and 32.3\% respectively $(\mathrm{p}=0.001)$.
\end{abstract}

Conclusion: Statin Pretreatment reduces the occurrence of post-operative delirium after coronary artery bypass surgery.

Keywords: Coronary artery bypass surgery, Cardiopulmonary bypasses, Post-operative delirium, Statin, Confusion Assessment Method

\section{Note:}

This article was written using the STROBE Statement-Checklist of items that should be included in reports of cohort studies.

\section{Corresponding author:}

Assistant Professor Dr. Behnam Askari, Department of Cardiovascular Surgery, Faculty of Medicine, Urmia University of Medical Sciences, Urmia, Iran.

Tel: +98.32375911, Fax: +98.32375917, Email: behnamaskar@yahoo.com

Received: August 02, 2018, Accepted: September 15, 2018, Published: November, 2018

iThenticate screening: September 09, 2018, English editing: October 02, 2018, Quality control: October 03, 2018

This article has been reviewed / commented by four experts

Ethics approval: IR.UMSU.REC.1396.273 (Urmia University of Medical Sciences)

(C) 2018 The Authors. This is an open access article under the terms of the Creative Commons Attribution-NonCommercialNoDerivs License, which permits use and distribution in any medium, provided the original work is properly cited, the use is non-commercial and no modifications or adaptations are made. 


\section{Introduction}

Despite much progress in cardiac surgery and anesthesia techniques, delirium after coronary artery bypass grafting (CABG) remains a common complication (1). Delirium is a degradation of brain activity defined by fluctuating mental status with changes in consciousness, attention, cognition, and perception, and is classified into three subtypes: 1) hyperactive, 2) hypoactive, and 3) mixed. Hyperactive delirium is determined by active symptoms such as agitation and restlessness, while hypoactive delirium is accompanied by unresponsiveness and motionlessness (2, 3 ). The incidence of post-operative delirium (POD) varies between studies, ranging from $1 \%$ to $50 \%$ (4). Delirium is associated with increased morbidity and mortality, prolonged stay in intensive care unit (ICU), prolonged hospital stay, and increased health care cost $(4,5)$. The definitive etiology and pathogenesis of delirium after cardiac surgery is unclear but it is likely to be complex and multifactorial, resulting from interaction of numerous predisposing and precipitating factors. Among these factors, older age, history of stroke and peripheral vascular disease are very important for interaction with increased cerebral atherosclerosis $(6,7)$. On the other hand, neuroinflammation is one the most causative hypotheses related to POD manifestation (8). According to Stone NJ et al. (9) and Katznelson R et al. (10), low-density lipoprotein (LDL), total cholesterol levels, and the risk of adverse cardiovascular events decrease by administration of statins or 3-hydroxy-3-methylglutaryl coenzyme A (HMG-CoA) inhibitors. Statin administration has been shown to decrease morbidity and mortality after cardiac and major non-cardiac surgery (10, 11). Statins or 3-hydroxy-3-methylglutaryl coenzyme A reductase inhibitors are powerful lipid-lowering medications and its administration can decrease the risk of cardiovascular adverse outcomes. Furthermore, several studies have demonstrated that statin therapy reduces morbidity and mortality in cardiac and non-cardiac operations $(10,11)$.

Several experimental clinical investigations have shown that statins can improve perioperative central neuronal protection. Creation of these effects by statins is beyond their lipid lowering activity, so-called cholesterolindependent or 'pleiotropic effect' including anti-inflammatory, anti-thrombogenic and immunomodulatory properties (12). Whereas statin pretreatment is associated with a reduction of POD in patients undergoing cardiac surgery (10), there is some controversy related to this protective effect of statin on delirium following cardiac surgeries $(13,14)$. According to clinical results, the effects of statins on delirium after cardiac surgery is limited and controversial, and based on our knowledge, there was no study in Iran to determine the effect of statin on delirium. Therefore, our study was aimed to determine whether pre-operative use of statins is associated with decreased delirium rates in patients undergoing coronary artery bypass grafting ( $\mathrm{CABG}$ ) with $\mathrm{CPB}$ (cardiopulmonary bypass).

\section{Material and Methods}

\subsection{Study design and participants}

This prospective cohort study enrolled patients from June 2017 through April 2018. The study was conducted in an Iranian governmental educational, heart center hospital (Seyedoshohada Hospital, Urmia, Iran). The reason for choosing this hospital was that it is the referral center in West Azerbaijan Province. Patients were selected based on receiving statins $10 \mathrm{mg}$ or $20 \mathrm{mg}$ in the pre-operative period for at least 1 month, whereas Group B patients had not been receiving statin before admission to hospital because of reasons as decided by the primary physician. Two hundred patients participated in each group.

\subsection{Selection criteria}

\subsubsection{Inclusion criteria}

The inclusion criteria were patients who were candidate for isolated elective CABG with $\mathrm{CPB}$ under general anesthesia.

\subsubsection{Exclusion criteria}

The exclusion criteria were the following: preexisting cerebrovascular disease, psychiatric illness, left ventricular ejection fraction $<30 \%$, severe left main stenosis, and redo or emergency CABG surgery, patients with hepatic impairment (aspartate aminotransferase or alanine aminotransferase $>$ twice the normal limit), renal disease (creatinine $>2 \mathrm{mg} / \mathrm{dl}$ ).

\subsection{Exposure and outcomes}

\subsubsection{Exposure}

The exposures in this study were defined as taking statins before surgery. Two hundred patients were selected randomly based on the exposure of interest. Group A patients had been receiving atorvastatin $10 \mathrm{mg}$ or $20 \mathrm{mg}$ in the pre-operative period for at least 1 month, whereas Group B patients had not been receiving statins before admission to hospital because of some reason as decided by the primary physician. 


\subsubsection{Outcomes}

The primary outcome was defined as the POD occurrence in each group. Delirium assessment was performed according to the Confusion Assessment Method (CAM) every 8 hours until discharge from ICU, by the ICU physician or the trained cardiovascular ICU nurses, who were not involved in the study. This assessment was based on the Diagnostic and Statistical Manual of Mental Disorders criteria and it included four stages: (1) an acute onset of changes or fluctuations in the course of mental status, (2) inattention, (3) disorganized thinking, and (4) an altered level of consciousness. A patient who demonstrated features 1 and 2 in addition to either feature 3 or 4 was defined as delirious. Patients were considered as having delirium if they experienced it on at least two positive calculations during ICU admission

\subsection{Procedure}

Patients, before undergoing CABG, may have met with two different approaches by clinicians; administrating of statins vs. not receiving statins. Participants in this study were selected among each of two approaches. Therefore, Group A ( $=200)$ (exposure group) received statins $10 \mathrm{mg}$ or $20 \mathrm{mg}$ in the pre-operative period and Group B $(n=200)$ had not received statins before admission to hospital. Anesthetic management and surgical techniques were the same for all patients. Oral lorazepam (1-2 mg) was administrated for all patients 1 to $2 \mathrm{~h}$ before surgery. All demographic and underlying diseases data including age, sex, NYHA (New York Heart Association) class, history of diabetes, hypertension, anemia ( $\mathrm{Hb}<12 \mathrm{~g} / \mathrm{dl}$ ), cerebrovascular disease and transient ischemic attack (TIA) were collected. Anesthesia was induced with intravenous thiopental sodium (100-150 mg/kg), sufentanil (1-2 $\mu \mathrm{g} / \mathrm{kg})$, midazolam $(0.1 \mathrm{mg} / \mathrm{kg})$ and trachea was intubated after cisatracurium $(0.15-0.20 \mathrm{mg} / \mathrm{kg})$ and maintained with TIVA (total intravenous anesthesia), infusion dose propofol $(100 \mu / \mathrm{kg} / \mathrm{min})$, sufentanil $(50-100 \mu / \mathrm{kg} / \mathrm{h})$, and cisatracurium $(5 \mu / \mathrm{kg} / \mathrm{min})$ and $\mathrm{O}_{2}$ in air (1:1). Monitoring including electrocardiogram, arterial blood pressure, pulse oximetry, central venous pressure, end-tidal $\mathrm{CO}_{2}$, BIS (bispectral index), and were used throughout the surgery period. Systemic anticoagulation was achieved with intravenous heparin $300 \mathrm{IU} / \mathrm{kg}$ with a target (Activated clotting time) ACT $\geq 480$ second. Management of CPB included systemic temperature $30-32{ }^{\circ} \mathrm{C}$, a stat pH management, targeted mean arterial pressures (MAP) between 50-70 $\mathrm{mmHg}$, Myocardial protection was achieved with intermittent antegrade, and occasionally retrograde, blood cardioplegia. Furthermore, duration of CPB, Anemia: Hematocrit $(\mathrm{HCT})<19$, Mean arterial pressure (MAP) $<50 \mathrm{mmHg}$ (hypotension during CPB), massive transfusion (blood transfusion $\geq 5$ units) were recorded. After separation from CPB, heparin was neutralized with 1 mg protamine per 100 IU of heparin, to achieve an activated clotting time within $10 \%$ of baseline. After end of surgery, patients were extubated by the ICU physician based on the following criteria: patient consciousness and cooperation, arterial oxygen saturation $\geq 95 \%$ with inspired oxygen fraction $\leq 50 \%$, hemodynamic stability, no excessive bleeding, and absence of uncontrolled arrhythmia. Patients in the statins group resumed taking statins after the second day. No patients received any other medication such as steroid or Non-steroid anti-inflammatory drugs (NSAIDs).

\subsection{Statistical Analysis, bias, and confounders}

In descriptive analysis, we determined frequency of numeric variables and mean \pm SD (standard deviation) for quantitative variables. Kolmogorov Smirnov test was used to detect the normal distribution of data. In order to compare the frequency of delirium among patients who used statin vs. those who did not, we applied Chi Square test. Independent samples t-test and Fisher exact test were subsequently used to compare quantitative and qualitative variables between the two groups. Selection bias was one of the biases in this study, by using stratified analysis we reported the result of the study in each stratification (age, sex). Some confounder variables such as hypertension, diabetes, and length of stay were adjusted by using logistic regression analysis. Logistic regression analysis was performed to determine the effect of covariates on the outcome (delirium). First, in the unadjusted model, the association of using statins and delirium was evaluated. The regression model was then adjusted by demographic and underlying diseases (sex, age, diabetes, and hypertension) and clinical variables. The significant level was considered $<0.05$. In order to avoid selection bias, the two groups were analyzed by age and sex. We also used logistic regression analysis to adjust confounding variables.

\subsection{Research ethics}

Declaration of Helsinki - ethical principles for medical research involving human subjects (adopted by the $18^{\text {th }}$ General Assembly of the World Medical Association [WMA], Helsinki, Finland, June 1964 and last amended by the $64^{\text {th }}$ WMA General Assembly, Fortaleza, Brazil, October 2013) was considered throughout the designing of the study protocol and its implementation. The institutional board and research ethics committee of Urmia University of Medical Sciences approved the study (Ref: IR.UMSU.REC.1396.273). The aim of the study was explained to participants before they entered the study. To ensure that all participants were aware of the procedures, an 
http://www.ephysician.ir

information sheet about the goal and nature of the study, its objectives, and the procedure of the study and potential risks was given to the participants to read before they signed the written informed consent form.

\section{Results}

All patients included in the study underwent CAM-ICU assessment every 8 hours during their cardiovascular ICU stay. There was no difference regarding demographic parameters (Table 1). POD was present in 14.2\% of patients (57 out of 400) at any time during their ICU stay. Nine patients (4.5\%) in Group A and forty-eight patients (24\%) in Group B had post-operative delirium. There was a significant difference in the occurrence of delirium across the two groups $(\mathrm{K}=15, \mathrm{p}=0.001)$. Demographic data and operative characteristics in patients of the two groups are demonstrated in Table1. Average of NYHA class was significantly higher in patients in Group B compared to patients in Group A (2.93 \pm 0.65 vs. $1.94 \pm 0.66, \mathrm{p}=0.01)$. CPB duration was significantly higher in Group B compared to Group A (127.17 \pm 44.34 vs. $114 \pm 38.44, \mathrm{p}=0.002)$. Similarly, Aorta cross clamp length was significantly longer in Group B than group A, $(92.41 \pm 49.83$ vs. $77.49 \pm 40.30, \mathrm{p}=0.001)$. Duration of post-operative mechanical ventilation was significantly prolonged in Group B (24.03 \pm 9.39 vs. $21.48 \pm 7.87, p=0.003)$. Moreover, the duration of ICU stay

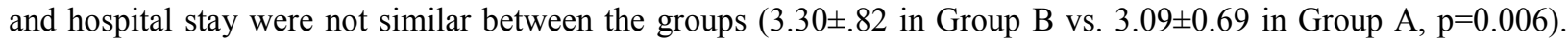
There was no post-operative mortality. Incidence of POD in patients $\geq 60$ years in Group A was significantly lower than in the patients in Group $\mathrm{B}, 4.2 \%$ and $32.3 \%$ respectively $(\mathrm{p}=0.001)$. Univariate analysis showed that administration of statins was associated with reduced post-operative delirium rates in patients $\geq 60$ years of age (Table 2).

Logistic regression analysis showed that patients who received statins were less likely to have delirium (OR: 0.14 , CI 95\%: 0.1-0.27, $\mathrm{p}=0.01)$. In adjusted model for demographic and clinical covariates, there was still similar association between the two groups, and the occurrence of delirium. In order to adjust confounder variables we applied logistic regression analysis, which detected the association of statins with less likely occurrence of delirium.

Table 1. Demographic data and operative characteristics of patients

\begin{tabular}{|l|l|l|l|l|}
\hline Variables & $\begin{array}{l}\text { Groups A (Statin), } \\
(\mathrm{n}=200)\end{array}$ & $\begin{array}{l}\text { Group B (No statin), } \\
(\mathrm{n}=200)\end{array}$ & p-value \\
\hline \multirow{5}{*}{ Pre-operative } & Age (year) & $62.04 \pm 10.85$ & $63.96 \pm 9.95$ & $0.06^{*}$ \\
\cline { 2 - 5 } & Male / Female & $132 / 68$ & $133 / 67$ & $0.91^{* *}$ \\
\cline { 2 - 5 } & Hb<12 gr/dl & $42(39.6 \%)$ & $64(60.4 \%)$ & $0.009^{* *}$ \\
\cline { 2 - 5 } & NYHA (class) & $1.94 \pm 0.66$ & $2.93 \pm 0.65$ & $0.01^{*}$ \\
\cline { 2 - 5 } & DM & $91(45.5 \%)$ & $81(40.5 \%)$ & $0.18^{* *}$ \\
\cline { 2 - 5 } & Hypertension & $126(63 \%)$ & $131(65.5 \%)$ & $0.33^{* *}$ \\
\cline { 2 - 5 } & CVA, TIA & $9(75 \%)$ & $3(25 \%)$ & $0.07^{* *}$ \\
\hline \multirow{5}{*}{ Intra-operative } & CPB time (minute) & $114.14 \pm 38.44$ & $127.17 \pm 44.34$ & $0.002^{*}$ \\
\cline { 2 - 5 } & ACC time (minute) & $77.49 \pm 40.30$ & $92.41 \pm 49.83$ & $0.001^{*}$ \\
\cline { 2 - 5 } & Surgery Duration (hour) & $6.26 \pm 1.40$ & $6.52 \pm 1.38$ & $0.06^{*}$ \\
\cline { 2 - 5 } & RBC > 5 unit & $0(0 \%)$ & $1(100 \%)$ & $0.34^{* *}$ \\
\cline { 2 - 5 } & RBC (number) & $1.88 \pm 0.93$ & $20.03 \pm 0.89$ & $0.001^{*}$ \\
\hline \multirow{5}{*}{ Post-Operative } & ICU length stay (day) & $3.09 \pm 0.69$ & $3.30 \pm 0.82$ & $0.006^{*}$ \\
\cline { 2 - 5 } & Mechanical Ventilation Time (hour) & $21.48 \pm 7.87$ & $24.03 \pm 9.39$ & $0.003^{*}$ \\
\cline { 2 - 5 } & Delirium & $9(4.5 \%)$ & $48(24 \%)$ & $0.001^{* *}$ \\
\hline
\end{tabular}

Continuous data are expressed as mean $\pm \mathrm{SD}$ and categorical data are expressed as number (\%). ACC: aortic cross clamp, CPB: cardiopulmonary bypass, CVA: cerebrovascular accident, DM: diabetes mellitus, Hb: hemoglobin, NYHA: New York Heart Association, RBC: red blood cells, TIA: transient ischemic attack.

* Independent-samples t-test, ** Chi-square test

Table 2. Comparison of the frequency of delirium based on age stratification among those receiving statin

\begin{tabular}{|l|l|l|l|l|l|l|}
\hline \multicolumn{2}{|c|}{} & \multicolumn{2}{|l|}{ Delirium } & Frequency & \multirow{2}{*}{$\mathrm{X}^{2 *}$} & \multirow{2}{*}{ p-value } \\
\cline { 2 - 6 } \multicolumn{2}{|c|}{} & Yes & No & & & \\
\hline \multirow{3}{*}{ Age (year) } & $\geq 60$ & 5 & 113 & 4.2 & \multirow{2}{*}{0.04} & \multirow{2}{*}{0.83} \\
\cline { 2 - 5 } & $<60$ & 4 & 78 & 4.9 & & \\
\cline { 2 - 5 } & Total & 9 & 191 & 4.5 & & \\
\hline
\end{tabular}




\section{Discussion}

The aim of this study was to determine the association between pre-operative statin administration and rate of POD in patients undergoing CABG. In this study, incidence of delirium in patients who were receiving pre-operative statins was significantly lower than in patients who were not treated with statins, and there was significant association between pre-operative statin therapy and reduced delirium rate. Some studies such as those by Kazmierski et al. (4) and Katznelson et al. (10) demonstrated similar results; as they found that perioperative therapy with statins was associated with decreased delirium rates in patients after cardiac surgery. In their study, postoperative delirium rate was reported as $8.4-11.5 \%$ and $11.5 \%$ respectively $(4,10)$. Since, in our study the number of patients was less than in other research, the discrepancy in the incidences of POD may be related to patient population. On the other hand, other studies were performed retrospectively whereas our study was performed prospectively.

In the present research, the incidence of POD in patients $\geq 60$ years with or without pre-operative statin therapy were $4.2 \%$ and $32.3 \%$ respectively, which suggests statins have considerable effect in decreasing POD rate in patients $\geq 60$ years. To explain this, the literature shows that ageing has been known as a factor to increase up to four-fold cytokines level and acute phase proteins (16). The increasing of inflammatory response in the elderly may explain the reason why the anti-inflammatory and anti-thrombotic effects of statins are particularly prominent in this patient population (17).

Among numerous hypotheses on the pathophysiology of POD, the most recent hypothesis involves neuroinflammation (18). Cardiac operation with CPB is associated with a profound systemic inflammatory response by reason of surgical trauma, and the contact between blood and synthetic circuit surfaces promote the blood-barrier dysfunction, cerebral inflammation, and glial cell injury, and finally lead to POD (19, 20). In recent times, neuroinflammation has been the most common theory for the explanation of pathophysiology of POD (18). Onpump cardiac surgery created serious systemic inflammatory reactions due to interaction among blood and artificial circuit surface, surgical trauma that induces blood-brain barrier deterioration, brain inflammation and glial cell damage and ultimately POD $(19,20)$. However, in this study, duration of CPB and ACCC time in patients in Group A was shorter than in patients in Group B, which may suggest the lesser effect of using statins on delirium by interfering with these covariates.

Although the exact mechanism of POD reduction by statins is not clear, it has been recommended that the neuroprotective effects of statins are due to either lipid lowering capability or the complex effects on inflammation and endothelial function (21). It is known that short or long-term administration of statin therapy in many kinds of cerebral damage can decrease the extent of the infarct area and increase cerebral blood flow in the ischemic region, leading to enhanced behavioral functions $(22,23)$. Furthermore, statins could reduce the permeability of the human blood-brain barrier and limit the transmigration of leukocytes. Several clinical studies have shown that statins, by affecting adhesion molecules, chemoattractant proteins, proinflammatory transcription factors, and proinflammatory enzymes, prevent inflammatory processes. They corrected endothelial function, firmed plaques, and stimulated neovascularization $(24,25)$.

In this study, patients who were not treated with statins had longer mechanical ventilation time and ICU stay compared to patients in Group A and we found statins administration, by decreasing POD and preventing some postoperation morbidity, may lead to change in health care costs. Mariscalco et al. (13), and Mathew et al. (14) demonstrated inconsistent results compared to our study, as they found that the pre-operative statins were not associated with a decreased rate of delirium in patients undergoing CABG surgery. A logical explanation for such difference is that the design and sample size of their studies were different from the present study. Almost $50 \%$ of patients in our study received atorvastatin versus $39 \%$ of patients in the study by Mariscalco and colleagues and only $22 \%$ of patients in the study by Mathew et al. Their study was retrospective, whereas our study was prospective and in current study assessment, statins on POD was accomplished in the early post-operative period, while Matthew et al. (14) evaluated cognitive function 6 weeks after surgery. On the other hand, there is a possibility that POD symptoms have been confounded by post-operative cognition dysfunction. According to Cerejeira $\mathrm{J}$ et al., the lack of statin protection could certainly be related to the complex multifactorial pathogenesis of post-operative delirium (8). They believed that changes in catecholamine levels after CPB use, cerebral embolization detected during the surgical procedure, decreased cardiac output observed after the operation, and prolonged ICU stay may be superior in the protective effects of pre-operative statins on delirium (4, 5-7, 26, 27). Likewise, Cruz JN et al., in a similar prospective study, obtained comparable results. The use of pre-operative statins is not correlated with post-operative 
delirium in patients undergoing cardiac surgery. They supposed that statins' systemic effects and pleiotropic effects, can differ between different drugs; this difference could indirectly influence neurological findings (28). The absence of protective effects of statins may be associated to several complicated underlying causes of pathogenesis of postoperative delirium (8). It has been assumed that change in post CPB catecholamine levels, intra-operative cerebral emboli, post-operative low cardiac output and lengthened ICU stay may be more plausible rather than the effect of statins on occurrence of delirium. $(4,5-7,26,27)$. Similarly, Cruz JN et al., through a similar prospective study, found that pre-operative statin therapy is not related to manifestation of delirium after cardiac operation. They believed that systemic and pleiotropic effects of statins may be different between various types of statin drugs, and this difference can indirectly influence neurologic results (28).

\section{Limitations and strength of study}

The prospective nature of a cohort study made this study as the strongest observational study. This study had several limitations. First, it was an observational study, which is prone to some biases; however, as it is described in the materials and methods section, we considered measures to avoid selection bias. Second, it was accomplished during a short period and on a small population of patients, while a larger sample size is required, to reach better outcomes. Third, our findings are based on association rather than a causative relationship among statin therapy and decreased post-operative delirium rates. Consequently, a double-blind, randomized, placebo-controlled trial is recommended to validate these findings. Last, the absence of delirium determination after ICU discharge could lead to an underestimation of its incidence.

\section{Conclusions}

This study found that the occurrence of delirium is less likely among those who received statins, even after adjusting for confounding variables. However, this association has not been detected among patients less than 60 years of age in stratified analysis. Small sample size might be the reason that we could not find this association among younger adults. Further study with a stronger design such as clinical trials with a larger sample size may be needed to prove this association.

\section{Acknowledgments:}

We are grateful to our ICU colleagues for their cooperation and interest in assessment of patients during the study period in the cardiovascular ICU Seyedoshohada Heart Center, affiliated to the Urmia University of Medical Sciences, in Urmia, Iran.

\section{Funding:}

The authors confirm that this study was not granted or funded by any institution.

\section{Conflict of Interest:}

There is no conflict of interest to be declared.

\section{Authors' contributions:}

All authors contributed to this project and article equally. All authors read and approved the final manuscript.

\section{References:}

1) Funder KS, Steinmetz J, Rasmussen LS. Cognitive dysfunction after cardiovascular surgery. Minerva Anestesiol. 2009; 75(5): 329-32. PMID: 19412153.

2) First MB. Diagnostic and statistical manual of mental disorders. 4th edition. Washington, DC: American Psychiatric Association Press; 2000: 136-9.

3) Liptzin B, Levkoff SE. An empirical study of delirium subtypes. Br J Psychiatry. 1992; 161: 843-5. doi: 10.1192/bjp.161.6.843. PMID: 1483173.

4) Kazmierski J, Kowman M, Banach M, Fendler W, Okonski P, Banys A. et al. Incidence and predictors of delirium after cardiac surgery: Results from The IPDACS Study. J Psychosom Res. 2010; 69(2): 179-85. doi: 10.1016/j.jpsychores.2010.02.009. PMID: 20624517.

5) Koster S, Hensens AG, van der Palen J. The long-term cognitive and functional outcomes of postoperative delirium after cardiac surgery. Ann Thorac Surg. 2009; 87: 1469-74. doi: 10.1016/j.athoracsur.2009.02.080. PMID: 19379886.

6) Trabold B, Metterlein T. Postoperative delirium: risk factors, prevention, and treatment. J Cardiothorac Vasc Anesth. 2014; 28: 1352-60. doi: 10.1053/j.jvca.2014.03.017. PMID: 25281048. 
7) Redelmeier DA, Thiruchelvam D, Daneman N. Delirium after elective surgery among elderly patients taking statins. CMAJ. 2008; 179: 645-52. doi: 10.1503/cmaj.080443. PMID: 18809895, PMCID: PMC2535740.

8) Cerejeira J, Firmino H, Vaz-Serra A, Mukaetova-Ladinska EB. The neuroinflammatory hypothesis of delirium. Acta Neuropathol. 2010; 119: 737-54. doi: 10.1007/s00401-010-0674-1. PMID: 20309566.

9) Stone NJ, Robinson JG, Lichtenstein AH, Bairey Merz CN, Blum CB, Eckel RH, et al. 2013 ACC/AHA guideline on the treatment of blood cholesterol to reduce atherosclerotic cardiovascular risk in adults: a report of the American College of Cardiology/ American Heart Association Task Force on Practice Guidelines. J Am Coll Cardiol. 2014; 63(25 Pt B): 2889-934. doi: 10.1016/j.jacc.2013.11.002. PMID: 24239923.

10) Katznelson R, Djaiani GN, Borger MA, Friedman Z, Abbey SE, Fedorko L, et al. Preoperative use of statins is associated with reduced early delirium rates after cardiac operations. Anesthesiology. 2009; 110: 67-73. doi: 10.1097/ALN.0b013e318190b4d9. PMID: 19104172.

11) Kennedy J, Quan H, Buchan AM, Ghali WA, Feasby TE. Statins are associated with better outcomes after carotid endarterectomy in symptomatic patients. Stroke. 2005; 36: 2072-6. doi: 10.1161/01.STR.0000183623.28144.32. PMID: 16179564.

12) Profumo E, Buttari B, Saso L, Rigano R. Pleiotropic effects of statins in atherosclerotic disease: focus on the antioxidant activity of atorvastatin. Curr Top Med Chem. 2014; 14: 2542-51. doi: 10.2174/1568026614666141203130324. PMID: 25478882.

13) Mariscalco G, Cottrini M, Zanobini M, Salis S, Dominici C, Banach M, et al. Preoperative statin therapy is not associated with a decrease in the incidence of delirium after cardiac operations. Ann Thorac Surg. 2012; 93: 1439-48. doi: 10.1016/j.athoracsur.2012.02.012. PMID: 22541176.

14) Mathew JP, Grocott HP, McCurdy JR, Ti LK, Davis RD, Laskowitz DT, et al. Preoperative statin therapy does not reduce cognitive dysfunction after cardiopulmonary bypass. J Cardiothorac Vasc Anesth. 2005; 19: 294-9. doi: 10.1053/j.jvca.2005.03.004. PMID: 16130053.

15) Ely EW, Gautam S, Margolin R, Francis J, May L, Speroff T, et al. The impact of delirium in the intensive care unit on hospital length of stay. Intensive Care Med. 2001; 27: 1892-900. doi: 10.1007/s00134-0011132-2. PMID: 11797025.

16) Krabbe KS, Pedersen M, Bruunsgaard H. Inflammatory mediators in the elderly. Exp Gerontol. 2004; 39(5): 687-99. doi: 10.1016/j.exger.2004.01.009. PMID: 15130663.

17) Yaffe K, Barrett-Connor E, Lin F, Grady D. Serum lipoprotein levels, statin use, and cognitive function in older women. Arch Neurol. 2002; 59: 378-84. PMID: 11890840.

18) Simone MJ, Tan ZS. The role of inflammation in the pathogenesis of delirium and dementia in older adults: a review. CNS Neurosci Ther. 2011; 17: 506-13. doi: 10.1111/j.1755-5949.2010.00173.x. PMID: 20553303.

19) Reinsfelt B, Ricksten SE, Zetterberg H, Blennow K, Fredén-Lindqvist J, Westerlind A. Cerebrospinal fluid markers of brain injury, inflammation, and blood-brain barrier dysfunction in cardiac surgery. Ann Thorac Surg. 2012; 94: 549-55. doi: 10.1016/j.athoracsur.2012.04.044. PMID: 22698770.

20) Newman MF, Mathew JP, Grocott HP, Mackensen GB, Monk T, Welsh-Bohmer KA, et al. Central nervous system injury associated with cardiac surgery. Lancet. 2006; 368: 694-703. doi: 10.1016/S01406736(06)69254-4. PMID: 16920475.

21) Vaughan CJ, Delanty N. Neuroprotective properties of statins in cerebral ischemia and stroke. Stroke. 1999; 30: 1969-73. PMID: 10471452.

22) Amarenco P, Labreuche J, Lavallee P, Touboul PJ. Statins in stroke Prevention and carotid atherosclerosis: Systematic review and up-to-date meta-analysis. Stroke. 2004; 35: 2902-9. doi: 10.1161/01.STR.0000147965.52712.fa. PMID: 15514180.

23) Stepien K, Tomaszewski M, Czuczwar SJ. Neuroprotective properties of statins. Pharmacol Rep. 2005; 57: 561-9. PMID: 16227638.

24) Ifergan I, Wosik K, Cayrol R, Kebir H, Auger C, Bernard M, et al. Prat A: Statins reduce human bloodbrain barrier permeability and restrict leukocyte migration: Relevance to multiple sclerosis. Ann Neurol. 2006; 60: 45-55. doi: 10.1002/ana.20875. PMID: 16729291.

25) Libby P, Aikawa M. Mechanisms of plaque stabilization with statins. Am J Cardiol. 2003; 91: 4B-8B. PMID: 12615292.

26) Koenig MA, Grega MA, Bailey MM, Pham LD, Zeger SL, Baumgartner WA. Statin use and neurologic morbidity after coronary artery bypass grafting. Neurology. 2009; 73(24): 2099-106. doi: 10.1212/WNL.0b013e3181c677f6. PMID: 19907012, PMCID: PMC2790220. 
27) Bucerius J, Gummert JF, Borger MA, Walther T, Doll N, Falk V, et al. Predictors of delirium after cardiac surgery delirium: effect of beating-heart (off-pump) surgery. J Thorac Cardiovasc Surg 2004; 127: 57-64. doi: 10.1016/S0022. PMID: 14752413.

28) Cruz JN, Tomasi CD, Alves SC, Macedo RC, Giombelli V, Cruz JG, et al. The incidence of delirium in patients pretreated with statins who remain in an intensive care unit after cardiac surgery. Rev Bras Ter Intensiva. 2012; 24(1): 52-7. doi: 10.1590/S0103-507X2012000100008. PMID: 23917713. 